\title{
Determination of Variables That Affect the Remission Rate of Sewing Operations in a Textile Company
}

\author{
Josefa Angelie Dilla Revilla, Leianne Yusi Casupang \\ University of the Philippines Los Baňos, Laguna, Philippines \\ Iris Ann Galarosa Martinez \\ University of the Philippines Diliman, Quezon City, Philippines \\ Ma. Laarni Dilla Revilla \\ Philippine Institute for Development Studies, Makati City, Philippines
}

\begin{abstract}
In operations management, the learning curve has been an effective tool in estimating operator performance. However, discontinuities in work disrupt the learning process and a phenomenon called remission or forgetting occurs, resulting in increased time of performing the task upon resumption of work. The study aims to identify variables that significantly affect the remission rate of sewing operations in a manufacturing setting. Four variables - length of stint 1 , percent Difference ${ }_{\text {at stint } 1}$, gender, and product family, were identified. Statistical analyses, such as paired $t$-test, correlation, regression, and analysis of variance (ANOVA) were conducted in order to observe the relationships between the dependent variable and independent variables. For the results of the first general regression, gender was found to be an insignificant variable in predicting remission rate, while product family, length of stint 1 , and percent Difference at stint $1_{1}$ were statistically significant. Moreover, the final general regression, which excluded the insignificant gender variable and considered the (regrouped) product families, revealed that product family, length of stint 1 , and percent Difference ${ }_{a t}$ stint 1 were still statistically significant. Length of stint 1 had a moderately positive correlation with remission rate, while percent Difference ${ }_{\text {at stint } 1}$ had a moderately negative correlation with remission rate. Also, percent Difference ${ }_{\text {at stint } 1}$ was the largest contributor to the remission rate model. In terms of $R^{2}$, the goodness-of-fit of the model is moderate. Finally, the model yielded an absolute error of $5.08 \%$, indicating a high accuracy in predicting remission rate.
\end{abstract}

Keywords: remission rate, learning remission model, learning curve, effects of work break, continuous performance, manufacturing

\section{Introduction}

It is a common notion that a worker who gets acquainted with the work she/he is doing is expected to get

Josefa Angelie Dilla Revilla, assistant professor, Department of Industrial Engineering, University of the Philippines Los Baňos, Laguna, Philippines.

Leianne Yusi Casupang, Department of Industrial Engineering, University of the Philippines Los Baňos, Laguna, Philippines.

Iris Ann Galarosa Martinez, Ph.D., associate professor, Department of Industrial Engineering and Operations Research, University of the Philippines, Diliman, Quezon City, Philippines.

Ma. Laarni Dilla Revilla, research associate, Philippine Institute for Development Studies, Makati City, Philippines.

Correspondence concerning this article should be addressed to Josefa Angelie Dilla Revilla, Department of Industrial Engineering, University of the Philippines Los Baňos, Laguna 4031, Philippines. 
better through time. This is known as the learning curve. In quantifiable terms, the time of performing the task decreases as the number of repetitions of performing a certain operation increases (due to increased task familiarity). The learning curve varies from industry to industry, and company to company, hence estimates must be developed for each organization (Heizer \& Render, 2011).

In the Philippines, the Herrera Law amended the Labor Code in 1989 to legalize contractualization, a method of employment that offers shorter duration and cheaper labor. Since then, it has been popular among businesses in the Philippines (Uy, 2014). In this system, workers are hired for a fixed period of time not greater than six months and free from benefits which regular counterparts were entitled of. Amidst controversies addressing contractualization being an anti-worker scheme, industries have greatly benefited from this employment system through higher profits and flexibility in manpower (Ocampo, 2014).

When the worker is relieved from work, either due to end of contract or transfer to another product line, she is usually rehired, since she already has the work familiarity and experience. This is in contrast to hiring new (untrained) workers, which will require training and familiarization of the task. This will reduce the training costs incurred by the company. In the case of a transferred worker to another operation or product line, after some time (e.g., when production of the product is continued), the worker is transferred back to her previous operation and/or product line.

This has been a common practice, especially now that there is a very tight global market competition where cost reduction is of great interest. However, a break from work would result in the decay of skill gained when working, known as learning remission or forgetting. Hancock and Bayha (1982) defined remission as a function of the operator's position on the learning curve. As one progresses through the learning curve, the expected remission decreases in case a work stoppage occurs. Hence, in theory, the processing time after the resumption of work would be longer than the processing time prior to the break. The extent of forgetting varies per individual and is subjected to different factors.

\section{Research Background}

Several studies on the learning curve and remission rate have been conducted. A study by Driskell, Willis, and Copper (1992) noted that overlearning has a significant effect on retention on a moderate level and that retention is greatly influenced by the task type and the retention period. In 1998, Summers, Gregory, Kelly, and Harland investigated the possibility of skill loss due to poor learning rather than just forgetting. If better training is enforced, the information will be stored better and thus recalling would be easier (as cited by Human Factors Integration Defence Technology Centre, 2007). Moreover, Lawani, Hare, and Cameron (2014) conducted a study on skill decay of wind turbine technicians in the use of rescue and evacuation device. It was observed that a decline in performance occurred during the 28th to 90th days upon acquisition. They suggested that the impact of adding training will result in a slower rate of forgetting regardless of whether it is a refresher or not. In terms of ability, it has been predicted and validated that higher ability individuals retain more than lower ability ones (Arthur, Winston, Stanush, \& McNelly, 1998).

In the Philippine case, a study of Revilla and Martinez (2014) investigated the effect of different variables (work break length, number of unique operations during the work break, frequency of performing similar operation during the work break, and length of complete rest) on the average processing time after the work break. The study considered a special case of work break wherein operators do something (i.e., same operation but on different product model and other operation on different product models) during the break instead of 
completely resting and doing nothing. It was found that remission was reduced, when the frequency of performing similar operation during the work break was increased. Another conclusion drawn from the study is that the three remaining variables do not affect the average processing time after the break. Furthermore, the coefficient of determination $\left(R^{2}\right)$ of the model of the average processing time after the work break is equal to $50.86 \%$, indicating that the goodness-of-fit is moderate. Thus, other variables must be investigated to increase the fit of this model. This led to the development of this study.

This study has utilized the last point before the break and first data point after the break unlike the previous study of Revilla and Martinez (2014) which used the average processing time to measure remission. Four new variables that affect remission are determined: length of stint 1 , percent Difference ${ }_{\text {at stint } 1}$, gender, and product family. These variables were gathered from different researches of the same nature.

The study generally aims to determine the effect of significant variables to the learning remission of sewing operators. A regression equation will be generated to forecast the expected performance of the operators upon resumption to work. It is important to note that no research has been conducted yet on the effect of gender on the learning and rates of forgetting in the industry, specifically in manufacturing involving sewing operations.

Investigating remission especially in the manufacturing setting is necessary, not only to observe and explain but also to control this phenomenon that will benefit the industries involved. The findings of this study shall help cultivate the labor force by providing the best labor practices in line with the company's economical standards.

\section{Learning Remission as a Function of Operator's Position in the Learning Curve}

It is an inevitable situation when one stops performing a task for a substantial period of time. Changes in the production process, introduction of new technology, and intermittent production on the same product due to periods of low and/or seasonal demand are just some of the reasons for the stoppage of work. These interruptions lead to discontinuities in the learning curve, which commonly is known as remission or forgetting (Yelle, 1979).

Hancock and Bayha (1982) modeled remission based on the power curve model as a function of the operator's position in the learning curve, when work (in the aircraft assembly process) stoppage occurs (Freivalds \& Niebel, 2009). Furthermore, it is given by equation (1):

$$
y=k+\frac{(k-s)(x-1)}{1-x_{s}}
$$

where $y$ is the cycle time; $k$ is the value of first cycle time; $x$ is the number of cycles or of units produced; $s$ is the standard time; and $x_{s}$ is the number of cycles to standard time.

\section{Learning Remission as a Skill Decay and Factors Associated With It}

Skill decay is the "loss or decay of trained or acquired skills (or knowledge) after periods of non-use". It is "the progressive deterioration of knowledge and skills when they are not used over extended periods of time" (Arthur et al., 1998, pp. 51-101).

The study on skill decay had been prevalent on military science. It has been of interest to observe the loss of skill of military personnel, when their acquired skills have been rendered unused for a period of time. 
According to Arthur et al. (1998), the major factors that affect decay and retention of skills over periods of non-use are:

- retention interval - the retention of skills is negatively associated with the length of retention interval, meaning that the longer periods of non-use show a decline in skill proficiency;

- degree of overlearning - additional training after reaching the threshold score would lead to overlearning, hence prevents the decay of skill (Arthur et al., 1998);

- task type (divided into four-physical and cognitive, open-looped and closed-looped, speed and accuracy, and natural and artificial) — emission varies depending on the type of task being performed (Arthur et al., 1998; Kim, Koubek, \& Ritter, 2007);

- condition of retrieval: similarity of original learning and retention testing contexts-loss of skill is minimized if the conditions upon retrieval are closely similar to the previous learning or experience (Arthur et al., 1998);

- instructional strategies and training methods - if better training is enforced, the information will be stored better and thus recalling them would be easier (Human Factors Integration Defence Technology Centre, 2007);

- individual differences-gender has different memory retention span (Association for Psychological Science, 2008).

The next sections of the paper are as follows: research organization, results and discussions, conclusions, and areas for further study.

\section{Research Organization}

\section{Scope and Limitations}

Information used in the study was limited on the previous records of completion times of the operators performing various sewing operations on different product lines at a company that produces baseball and hockey gloves, etc. Sewing operations were assumed to be $80 \%$ operator-paced and $20 \%$ machine-paced based on observations on the study of Revilla and Martinez (2014). These which require high level of attention were considered, since the skill and proficiency of the operator will mainly influence the processing time of an operation unlike operations which are predominantly machine-paced. Work break of less than six months was considered, since contractual operators follow this kind of labor system in the Philippines.

\section{Conceptual Framework}

Figure 1 shows the conceptual framework of the study. Continuous performance of an operator assigned to a specific operation and product model, ranging from one to six months was considered. A special type of work break wherein other activities are performed during the break instead of complete rest was considered (on the average of three months).

This study investigated the learning remission of operators subjected to variables identified to have an effect on the learning curve. These variables were categorized into three: performance-based, product-related, and individual-based variables. Performance-based variables include the average processing time of operation and processing time just before work break; product-related variables include the product group or family. Meanwhile, individual-based variables include length of stint 1 and gender. Using these identified variables, the remission rate can be predicted through a regression model. 


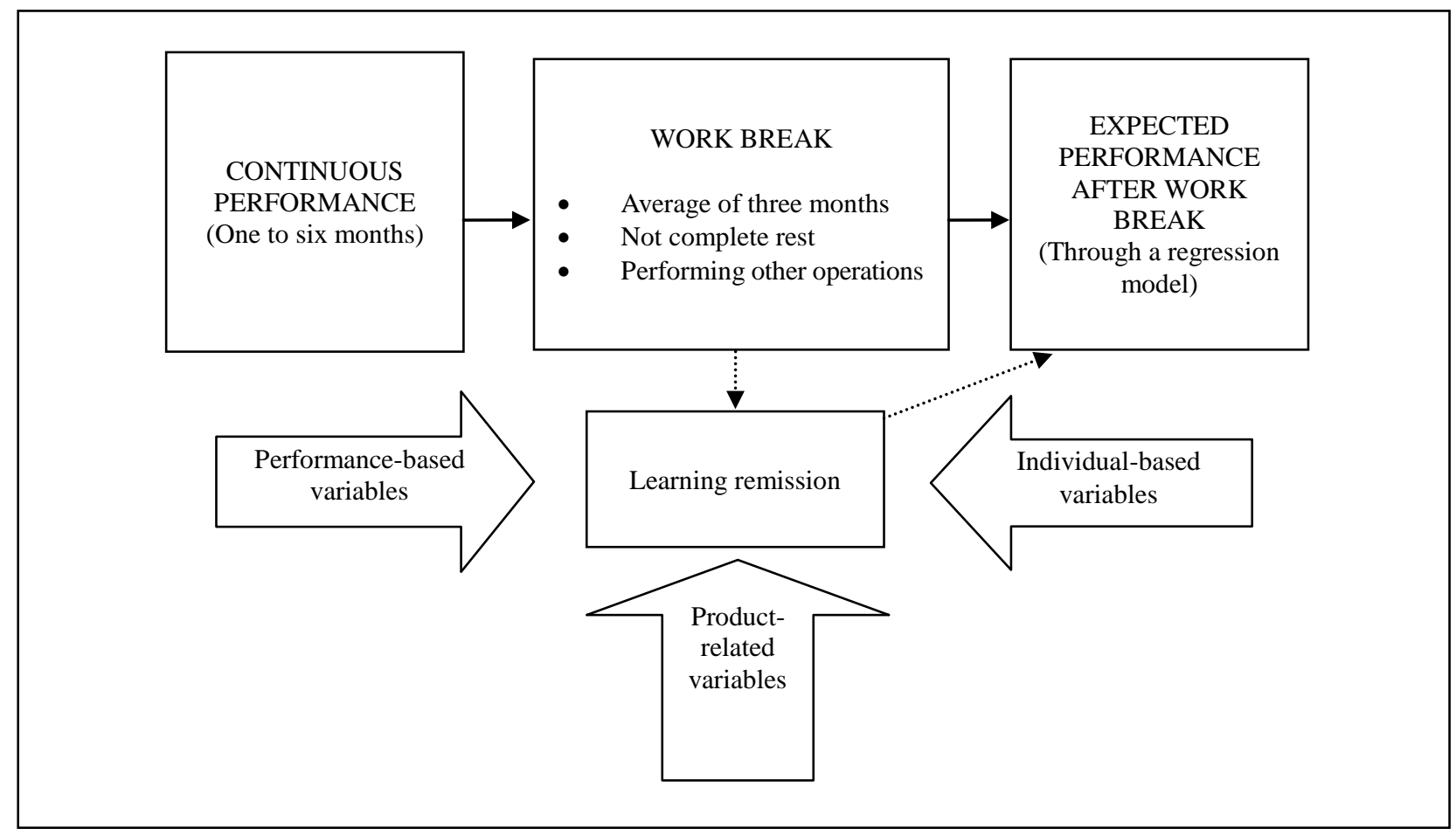

Figure 1. Conceptual framework of the research on the variables affecting learning remission.

\section{Definition of Variables}

Length of stint 1. Stint is simply the period in which the operator performed the operation in a specific product line. Stints that occurred before the work break were labeled as stint 1, while those that occurred after the break were labeled as stint 2 . This variable was considered as a predictor in the model to determine the effect of the length of stint 1 on the processing time after the work break.

Work break. The work break mentioned in this study does not mean total or complete rest. Operators perform other tasks, some of which were similar to their previous operation, and only differ in the product line.

Percent difference between the last point's processing time and the average processing time at stint 1. This was used to gauge the performance of the operator before she/he had taken the work break. The last data point of stint 1 and the first data point of stint 2 were considered, since the remission model of Hancock and Bayha (1982) considered one point only and not the average of data points, respectively. Also, remission is more evident when single data points are considered instead of average of data points wherein the data reach standardization. A positive percentage (increase) could mean a decrease in remission, which does not have an effect, or increase the remission of an operator after she/he has performed the same operation immediately after the work break. It is given by the formula:

$$
\% \text { Difference }_{\text {at stint } 1}=\left(\frac{P T \text { at last point }- \text { average } P T}{\text { average } P T}\right)_{\text {stint } 1} \times 100 \%
$$

where PT is the processing time (in minutes); \% Differenceat stint $1_{1}$ is the rate of change in processing time between the last point and the average PT of the first stint; PT at last point is the actual value of the last processing time before the work break; and average PT is the average of the actual processing times during the first stint. 
Product family. Each operator was ought to perform an operation on a specific product line for the entire stint. Product models were initially grouped into 12 families: AA, BB, CC, DD, EE, FF, GG, HH, II, JJ, KK, and LL. This was done to determine if product variety affects learning remission.

Operator's gender. Each operator was classified either as male or female to determine if remission varies across gender classification.

Remission rate. First, to determine the most appropriate data point model to use for the computation of remission rate, paired $t$-test was conducted. One-data point, two-data point, and three-data point model were used. The remission rate of the two-data point model was computed by first taking the average processing time of the last two-data points of stint 1 and also the first two-data points of stint 2 . The resulting processing times were then used to compute for the remission rate using equation (3). Same procedure was applied to the computation of remission rate of the three-data point model, only that three-data points were used instead of two. If the remission rates of the three models were found to be statistically equal, the model to be used has to be the one-data point model which was commonly used by other remission models (e.g., Hancock and Bayha's model), and not the average of data points. It is computed using the formula:

$$
\text { Remission rate }=\frac{P T \text { at first point of stint } 2-P T \text { at last point of stint } 1}{P T \text { at last point of stint } 1} \times 100 \%
$$

where remission rate is the rate of change in processing time between the last point of stint 1 and the first point of the next stint; $P T$ at first point of stint 2 is the actual value of the processing time after the work break; and $P T$ at last point of stint 1 is the actual value of the last processing time before the work break.

\section{Statistical Analyses}

Paired $t$-test. It is used to determine whether there is a significant difference between two observations. In the study's case, the remission rates of having one-data point, average of two points, or three points were investigated, if their difference is significant to cause statistically different results.

Correlation. After the response and independent variables were defined, each pair of variable and the response had been graphed through a fitted line plot to show any pattern or relationship that exists among them. For linear relationships, the degree of correlation between each pair of response and predictor was quantified through the Pearson correlation coefficient. The strength of the correlation coefficient was evaluated through a guideline found in Table 1. Using the generated $p$-values, the significance of the correlation was determined.

Table 1

Pearson Correlation Coefficient Strength Evaluation

\begin{tabular}{ll}
\hline Pearson correlation coefficient & Strength of correlation \\
\hline $0<|r|<0.3$ & Weak correlation \\
$0.3<|r|<0.7$ & Moderate correlation \\
$|r|>0.7$ & Strong correlation \\
\hline
\end{tabular}

Source: Retrieved from http://www.sjsu.edu/faculty/gerstman/StatPrimer/correlation.pdf.

Regression analysis. Before regression analysis can proceed, there are statistical assumptions that must be satisfied. These are: (a) Variables were measured at least in the interval scale; (b) linear relationship between the response variable and predictors was satisfied; (c) significant outliers were not included; (d) independence among observations must be met, that is, no predictors can be expressed as a linear combination of other 
predictors; (e) homoscedasticity — equal variances of errors along the line of best fit; and (f) residuals (or errors) are approximately normal (Retrieved from https://statistics.laerd.com/spss-tutorials/linear-regression-usingspss-statistics.php).

Analysis of variance (ANOVA). ANOVA was performed to determine the individual contribution of the variables to the model.

\section{Validation of the Model}

To determine how well the model can accurately predict the remission rate, validation was carried out. The measure used was percent error, which measures the absolute closeness of the predicted value to the actual value.

Using equation (3), the predicted remission rate for each data point was derived. From the predicted remission rate, processing time after the work break was computed by manipulating equation (3) into:

$$
P T \text { at first point of stint } 2=\frac{\text { Remission rate (predicted }) \times(P T \text { at last point of stint } 1)}{100 \%}+P T \text { at last point of stint } 1
$$

Percent error of each data point was taken and their average was computed. A measure, which is frequently used in forecasting, called the mean absolute percentage error (MAPE), was used to determine the overall error of the model. It is computed using the formula:

$$
\operatorname{MAPE}(\%)=\frac{1}{N} \sum_{n-1}^{N} \frac{\mid \text { Actual }- \text { Forcast } \mid}{\text { Actual }} \times 100 \%
$$

where MAPE is the mean absolute percentage error and measures the magnitude of the difference of the predicted from the actual value; forecast is the predicted remission rate computed using the model; and actual is the actual remission rate from the data.

\section{Results and Discussions}

\section{Comparison of Three Models (One-Data Point, Two-Data Point, and Three-Data Point Model)}

To reduce the possibility of observing "chance" occurrences, the remission rates using one-data point, two-data points, and three-data points were tested, if they are statistically different from each other. In the last two methods, the average of data points was taken before the remission rate was computed. Taking the average of data points reduces the possibility of chance occurrence. Also, taking the average can show how remission rate changes with the number of data points used.

Paired $t$-test was the statistical method used and results showed that the three are statistically equal. Therefore, any of the three methods of computation of remission rates can be used. In this case, one-data point was used in developing the remission rate model.

\section{Correlation Between the Response Variable and Each Predictor}

The first step was to graph each predictor versus the response variable, remission rate. In this way, possible correlation can be visually observed. Several line/curve fits were used: linear, quadratic, and cubic function. The $p$-values from each fit were then computed and the coefficient of the function with a significant $p$-value was selected.

Remission rate and length of stint 1. Based from the comparison of graphical results as well as the 
$p$-values of each fit, it was found that the relationship between the two variables is linear at a 0.05 level of significance. Moreover, a Pearson correlation coefficient of 0.315 indicates a moderately positive correlation between the two variables. With a $p$-value of 0.007 , the obtained result is taken to be statistically significant.

Figure 2 further presents that the difference in processing times before and after the work break increases as the length of stint 1 increases, indicating that remission is greater on longer stints. This result is contrary to the hypothesis that longer stints would mean greater experience on the task and therefore lesser remission would be observed. One major observation to explain such phenomenon is that longer stints have relatively shorter work break (referring to Figure 3). It was found on previous study of Revilla and Martinez (2014) that the special type of work break has an impact on the operator's performance after resumption of work, specifically, the frequency of doing similar operations. This could explain why positive relationship exists between length of stint 1 and remission rate.

Remission rate and \% Difference $_{\text {at stint } 1 .}$. The relationship between the two variables is linear at a 0.05 level of significance. Moreover, a correlation coefficient of -0.582 indicated a moderately negative correlation between the two variables. With a $p$-value of 0.000 , the obtained result is statistically significant.

Based on the fitted line plot (referring to Figure 4), the difference in processing times before and after the work break decreases as the difference in processing times at stint 1 increases, indicating that operators who deviated farther from the average processing time showed higher improvement in processing time (as suggested by lesser remission) upon resumption of original work. This simply means that by keeping the performance within the average processing time, there will be lesser remission observed.

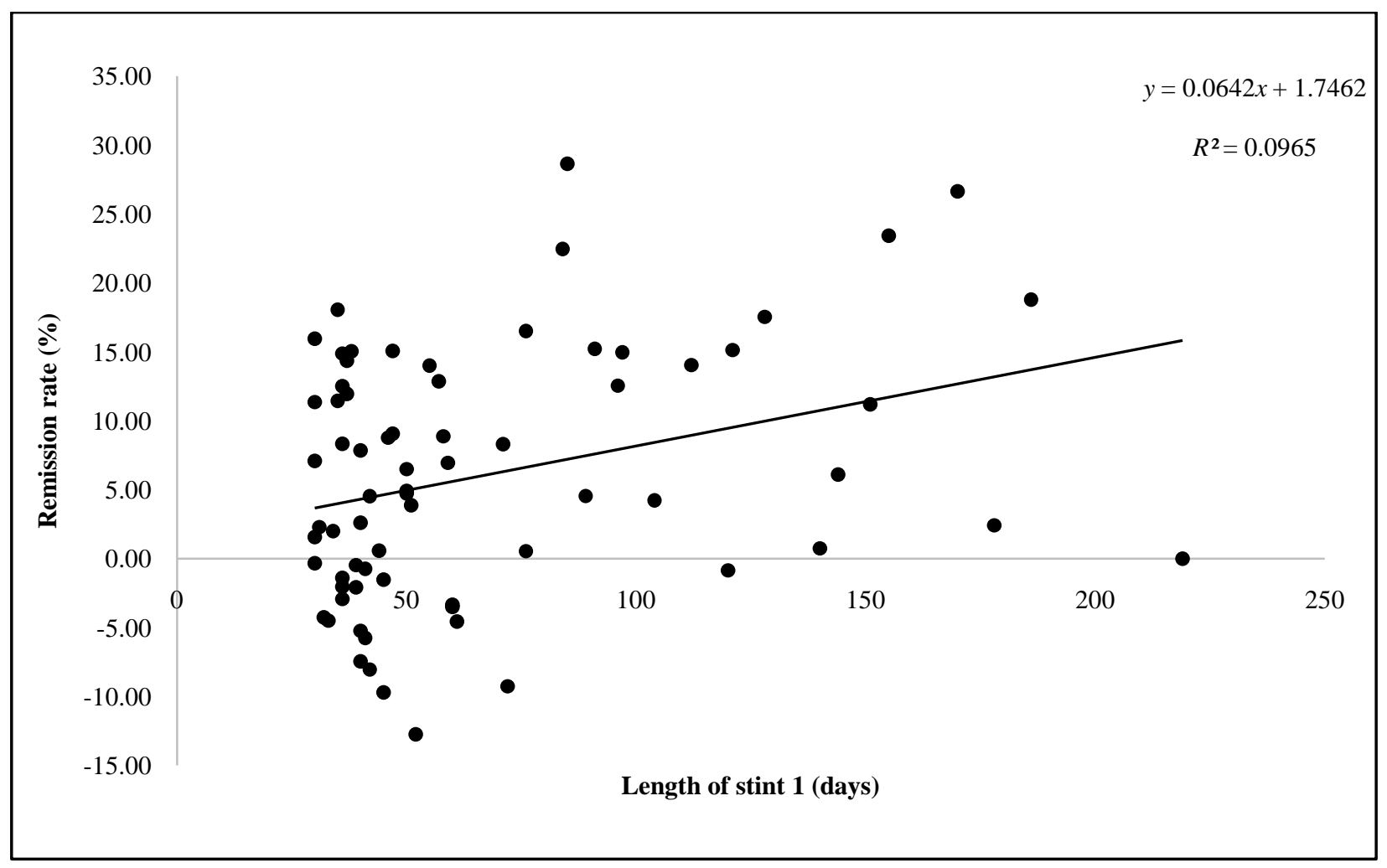

Figure 2. Fitted line plot of remission rate versus length of stint 1. 


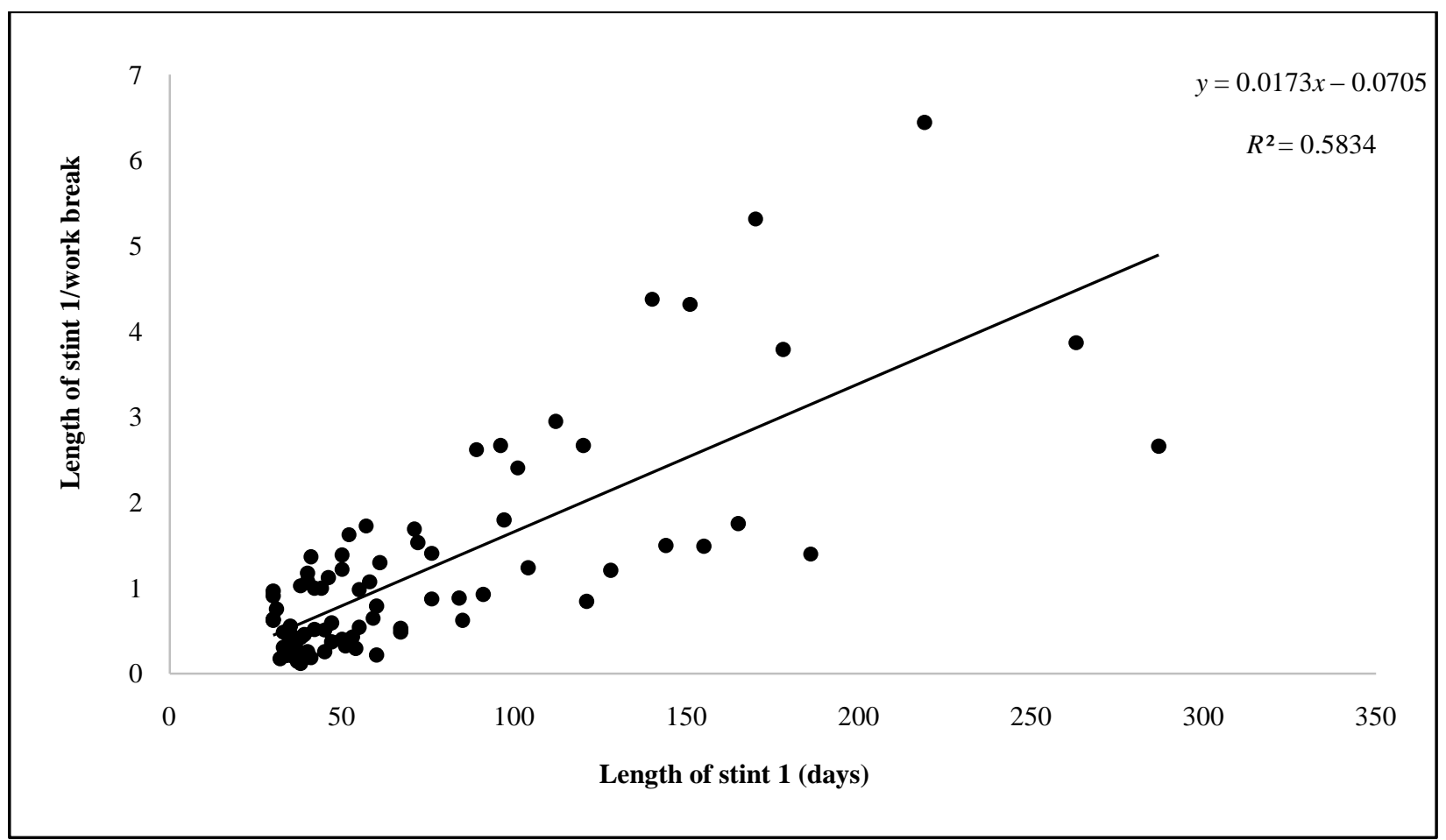

Figure 3. Fitted line plot of the length of stint 1 vs. length of stint 1/work break.

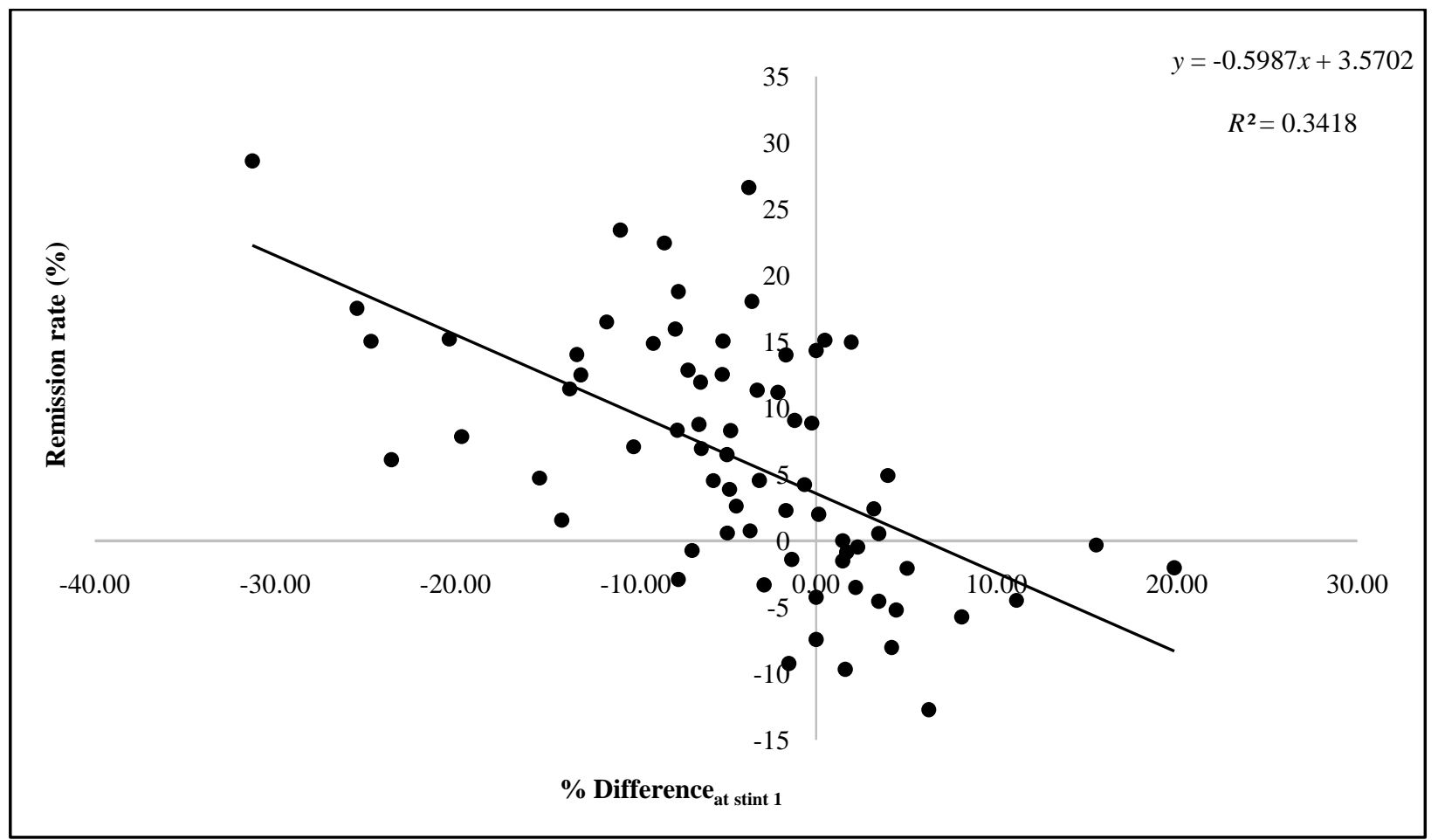

Figure 4. Fitted line plot of remission rate vs. \% Difference at stint $1 .^{1}$

\section{Regression Analysis Between the Response Variable and the Predictors}

The results in the previous section suggest the linear relationship of length of stint 1 and $\%$ Difference $_{\text {at stint }}$ ${ }_{1}$ to remission rate, hence regression analysis was possible. Additional assumptions mentioned earlier were also 
met. The result of the first general regression was performed to incorporate categorical variables into the model - product family and gender (referring to Table 2). It was observed that gender is not a significant variable in determining remission (at a 0.05 level of significance). This means that the remission rates of male and female operators are equal.

Table 2

ANOVA of the First General Regression Model

\begin{tabular}{|c|c|c|}
\hline Source & $p$-value & Result \\
\hline Gender & 0.618 & Not significant \\
\hline Product family & 0.022 & Significant \\
\hline Length of stint 1 & 0.033 & Significant \\
\hline$\%$ Difference $_{\text {at stint } 1}$ & 0.000 & Significant \\
\hline$R^{2}$ & & \\
\hline
\end{tabular}

All other variables were observed to be significant in determining remission. However, upon closely examining the $p$-values of the individual product families, it was found that some families are insignificant. For instance, only AA and GG were significant, while BB, CC, DD, EE, FF, HH, II, and LL were not. Therefore, grouping the insignificant families further was necessary to come up with a more valid model.

Ten out of the 12 product families were not significant and so they were grouped into a larger family denoted by XYZ, reducing the number of families into three. It was observed that only the constant in the generated equation varies given the product family.

\section{Result of the Final General Regression}

Since it was found that gender is not a significant variable in the model, it was removed from further analysis. Consequently, another general regression was performed, with product family as the remaining significant categorical variable (referring to Table 3). Upon regrouping the product family, the generated equations are summarized.

Table 3

\section{Linear Regression Equations}

\begin{tabular}{ll}
\hline Product family & Regression equation \\
\hline AA & $\%$ Difference $=-9.219+0.044$ Length of stint $1-0.670 \%$ Difference $_{\text {at stint } 1}$ \\
GG & $\%$ Difference $=3.053+0.044$ Length of stint $1-0.670 \%$ Difference $_{\text {at stint } 1}$ \\
XYZ & $\%$ Difference $=0.155+0.044$ Length of stint $1-0.670 \%$ Difference $_{\text {at stint } 1}$ \\
\hline
\end{tabular}

The coefficients of the variables - length of stint 1 and \% Difference at stint 1 are 0.044 and -0.670 , respectively. This means that for every one day increase in the length of stint 1 , an additional $0.044 \%$ in remission is expected. Meanwhile, for every one percent increase in the last point's processing time from the average processing time, a reduction of $0.670 \%$ in remission is expected.

To determine the adequacy of the model, $R^{2}$ was obtained to know the total variation in remission rate that is explained by the regression model (referring to Table 4). The results showed that $48.88 \%$ of the total variation in remission rate is explained by the regression model. There is no baseline of $R^{2}$ for one to say that the model is adequate or good. Since the model explained more than half of the variation in the response, it is a good model of the remission rate. The remaining $51.22 \%$ is associated with variables not included in the model and thus is subject for further study. 
Table 4

ANOVA of the Final General Regression

\begin{tabular}{|c|c|c|c|c|c|}
\hline Source & $d f$ & Seq SS & $F$ & $P$ & Conclusion (at $\alpha=0.05$ ) \\
\hline Product family & 2 & 147.63 & 6.035 & 0.004 & Significant \\
\hline Length of stint 1 & 1 & 506.17 & 5.509 & 0.022 & Significant \\
\hline$\%$ Difference $_{\text {at stint } 1}$ & 1 & $2,196.66$ & 48.63 & 0.000 & Significant \\
\hline Error & 66 & $2,981.07$ & & & \\
\hline Total & 70 & $5,831.52$ & & & \\
\hline$R^{2}$ & $48.88 \%$ & & & & \\
\hline
\end{tabular}

\section{Model Validation}

Validation was done to verify the ability of the model in predicting the remission rate accurately. The measure used was percent error, which measures the closeness of the predicted value to the actual value. Percent error of each data point was computed and their MAPE was taken.

Based from the computations, MAPE is equal to 5.08\%. This means that on the average, the predicted remission rate is $5.08 \%$ higher or lower than the actual.

\section{Conclusions}

Among the four variables considered in the study, only three have emerged to significantly determine the

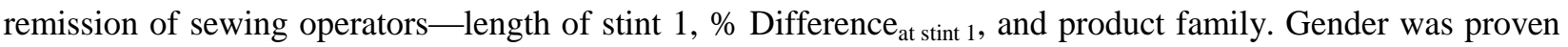
to have no significant effect on remission, considering this type of operation.

Overall, the regression model yielded a coefficient of determination $\left(R^{2}\right)$ of $48.88 \%$. The coefficients of the two variables - length of stint 1 and \% Difference $e_{\text {at stint } 1}$ are 0.044 and -0.670 , respectively; while the regrouped product family only affects the constants in each model. Moreover, the model yielded a MAPE of $5.08 \%$. The acceptable value of percent difference or error is $<10 \%$, therefore the model is good in terms of accurately predicting remission.

The results of the study may be used to adjust labor practices to further ensure employees' welfare and work efficiency. Specifically, as production and operations managers, it is important to predict the performance of the operator after a work break in order to adjust the level of production justifiable to the operator's start-up performance. Also, the scheduling decisions of firms may be improved based on the result that remission rates are associated with longer continuous performance. Additionally, since $\%$ Difference $_{\text {at stint } 1}$ was found to be a significant determinant of remission rate, the worker performance within the period of continuous performance should be maintained within the average to reduce the remission rate. Lastly, given that the type of product being processed affects the remission rate, firms must carefully assign workers on each product line in order to reduce the impact of switching product lines during the break on the remission rate.

The role of the learning curve and remission in predicting the performance level and its associated output level has been the main driving force in studying this area and applying it to real-life production systems.

\section{Areas for Further Study}

The study has enumerated several variables affecting remission of operators in various sewing operations in a leather goods manufacturing industry. However, there were still areas for further study.

- The length of stint 1 was the primary basis this study had considered as the experience of the worker. It would be best to know that the number of stints the operator had performed in the past, or his length of stay in 
the company. In this way, other indicators of experience can be counted in.

- The effect of age of the workers on remission. Past studies in other field of study have found the relationship of age and forgetting, given the common notion that a person easily forgets as she/he ages.

- It was found that only some of the product families have significant remission rates. However, reasons why they exhibit such behavior are of interest, thus a possible area for further study is needed.

\section{References}

Arthur, W., Winston, B., Stanush, P. L., \& McNelly, T. L. (1998). Factors that influence skill decay and retention: A quantitative review and analysis. Human Performance, 11(1), 57-101.

Association for Psychological Science. (2008). Sex differences in memory: Women better than men at remembering everyday events. Retrieved from www.sciencedaily.com/releases/2008/02/080220104244.htm

Driskell, J. E., Willis, R. P., \& Copper, C. (1992). Effect of overlearning on retention. Journal of Applied Psychology, 77(5), 615-622.

Freivalds, A., \& Niebel, B. (2009). Niebel's methods, standards and work design (12th ed.). New York, NY: McGraw-Hill Higher Education.

Gertsman, B. B. (n.d.). Correlation. Retrieved from http://www.sjsu.edu/faculty/gerstman/StatPrimer/correlation.pdf

Hancock, W. M., \& Bayha, F. H. (1982). Handbook of industrial engineering. New York: John Wiley and Sons.

Heizer, J., \& Render, B. (2011). Operations management (10th ed.). Upper Saddle River: Prentice-Hall Incorporated.

Human Factors Integration Defence Technology Centre. (2007). Literature review on skill fade. Retrieved from http://www.hfidtc.com/research/training/training-reports/phase-2/2-10-3-2-skill-fade.pdf

Kim, J. W., Koubek, R. J., \& Ritter, F. E. (2007). Investigation of procedural skills degradation from different modalities. In Proceedings from the 8th International Conference on Cognitive Modeling (pp. 255-260). Retrieved from http://acs.ist.psu.edu/papers/kimKR07.pdf

Laerd Statistics. (n.d.). Linear regression analysis using SPSS statistics. Retrieved from https://statistics.laerd.com/spss-tutorials/linear-regression-using-spss-statistics.php

Lawani, K., Hare, B., \& Cameron, I. (2014). Skill decay of wind turbine technicians in the use of rescue and evacuation device during emergency. Proceedings from CIB W099 International Conference Achieving Sustainable Construction Health and Safety (pp. 537-553). Retrieved from http://www.lth.se/fileadmin/healthsafety2014/Kenneth_Lawani__Billy_Hare.pdf

Ocampo, S. (2014). Labor contractualization: A 25-year bane to workers. Retrieved from http://www.philstar.com/opinion/2014/06/21/1337200/labor-contractualization-25-year-bane-workers

Revilla, J. A. D., \& Martinez, I. A. G. (2014). A comparative study of performance between operators with work discontinuity and without work discontinuity. Chinese Business Review, 13(5), 290-300.

Uy, V. (2014). Scrap Herrera Law which allowed “contractualization"-Labor research group. Retrieved from http://www.interaksyon.com/article/81890/scrap-herrera-law-which-allowed-contractualization---labor-research-group

Yelle, L. E. (1979). The learning curve: Historical review and comprehensive survey. Decision Sciences, 10(2), 302-328.

Summers, A., Gregory, D., Kelly, M., \& Harland, S. (1998). Predicting skill fade and procuring performance (UC). Dorchester, United Kingdom: Defence Evaluation and Research Agency. 